\title{
Novel MDR-reversing inhibitors of MRP subtypes related to antiretroviral drug resistance and CD4 cell counts
}

\author{
Andreas Hilgeroth*, Paul Naujoks, Sebastian Neuber, Marc Hemmer, Hermann Lage, Joséf Molnar \\ From International Symposium HIV and Emerging Infectious Diseases 2014 \\ Marseille, France. 21-23 May 2013
}

\section{Introduction}

Transmembrane efflux pumps play an increasing role in the success of ART. The occurrence of increasing mRNA levels of the efflux pump proteins P-gp and MRP subtypes has been associated with ART therapeutics like PIs and NNRTIs. Moreover, a relation has been suggested of MRP subtypes and CD4 cell counts. So there is a challenge to find selective MRP inhibitors which may reduce MRPinduced drug resistances as well as discussed effects on CD4 cells. We developed novel nonpeptidic inhibitors of important MRP subtypes related to both MRP-mediated resistances as well as reduced cell counts.

\section{Materials and methods}

The molecular structures of the various efflux pumps guided the development of both symmetric and nonsymmetric inhibitors dedicated to selectively inhibit the relevant MRP-subtypes. Inhibiting properties were evaluated in exclusively P-gp as well as MRP-overexpressing cell lines in comparison to nonexpressing parental cell lines with fluorescent substrates using flow cytometry.

\section{Results}

Our inhibitors with allover symmetric substitution patterns are excellent P-gp inhibitors, while a reduction of those symmetric elements concerning their number and exact positioning significantly lowers the P-gp inhibition whereas the MRP1 inhibition is found mainly increased. So selective inhibitors are demonstrated to influence the efflux pump type-related antiretroviral drug resistance. \section{Germany

Institute of Pharmacy, Martin Luther University Halle-Wittenberg, Halle, 